\title{
Learning dialogue with and without movement
}

\author{
HELGA NOICE \\ Elmhurst College, Elmhurst, Illinois \\ and \\ TONY NOICE \\ Indiana State University, Terre Haute, Indiana
}

\begin{abstract}
In two experiments, we investigated the contribution of physical movement to the processing and eventual retrieval of dramatic dialogue by untrained nonactors after they had received only brief procedural coaching. It was found that participants who processed a script by reading the text aloud while simultaneously moving in accordance with a director's instructions retained more material than did those who used verbal communication only or controls who deliberately memorized the same material. Furthermore, when the recall of the participants in the moving condition was analyzed on a speechby-speech basis, the results showed significantly greater memory for speeches that had been accompanied by movement than for speeches during which the same participant had remained in one place. These findings are discussed in the contexts of embodied cognition and multimodal memory models.
\end{abstract}

Previous studies have revealed that months after the final performance of a play, dialogue that had originally been accompanied by actors' movements about the stage was better recalled than dialogue that had been spoken when the actors had remained in one place (H. Noice \& T. Noice, 1999; H. Noice, T. Noice, \& Kennedy, 2000). This was true whether or not the actors duplicated the movements at test, suggesting that input-output similarity could not have been responsible for the results. In the present study, we investigated whether similar memory facilitation could be produced under experimental conditions with nonexpert participants who were given very brief exposure to the material.

The effects of movement on other types of text have been extensively investigated, most notably in connection with subject-performed tasks (SPTs). Researchers have frequently demonstrated that such phrases as "lift the cup" are better remembered when actually performed (with real or imaginary objects) than when acquired under standard verbal-learning instructions (e.g., Engelkamp, 1991; Kormi-Nouri \& Nilsson, 1998; for reviews, see Cohen, 1989; Engelkamp, 1998; Engelkamp \& Zimmer 1990, 1994; Nilsson, 2000). In many of these experiments, the SPT advantage appeared regardless of

Portions of Experiment 1 were reported at the 39th Annual Meeting of the Psychonomic Society, November 19-22, 1998, Dallas. Experiment 2 was supported by a grant to $H$. N. from the Faculty Development Fund of Elmhurst College. The authors thank Tricia Schopa and Megan Miller for their help in scoring the recall protocols. Thanks are also due Art Glenberg and an anonymous reviewer for their many helpful comments that greatly improved the paper. Correspondence concerning this article should be addressed to H. Noice, Department of Psychology, Elmhurst College, 190 Prospect Ave., Elmhurst, IL 60126 (e-mail: helgan@elmhurst.edu). whether or not movements were reenacted at test, the same result we found with theatrical scripts. However, in our experiments (H. Noice \& T. Noice, 1999; H. Noice et al., 2000), the recalled material was accompanied by movements that were not literally congruent with the verbal material. For example, in an SPT experiment, in order to learn the verbal phrase "cross my legs," the participant would be instructed to cross his or her legs. On the other hand, in a play, a character might sit down and cross his or her legs while saying "Don't bother about me, I'm perfectly comfortable here." Nevertheless, despite differences in procedure and the lack of a literal match between words and movement, actors' movements on-stage appear to enhance retention of accompanying verbal material in much the same way that literal movement does in SPT research. Of course, there are so many variations between SPT studies and our own, one could not say with any certainty that they are mediated by the same underlying mechanisms. For instance, in our studies, testing was done after delays of up to 5 months, whereas most SPT testing is done shortly after the learning phase. In addition, our participants were recalling lengthy connected discourse, whereas SPT experiments almost always use individual short verbal phrases.

Our research with professional actors has revealed a two-phase approach to their learning of roles (T. Noice \& H. Noice, 1997a). The first stage is analytical, involving extremely deep processing of the text into chunks, each chunk devoted to a separate intention of the character (H. Noice \& T. Noice, 1994). This, in itself, has been shown to enhance verbal memory (H. Noice \& T. Noice, 1993). However, for maximum effectiveness, these intentions must be processed, not just cognitively, but also motorically and emotively, a procedure we have termed active experiencing (T. Noice \& H. Noice, 1997a). For 
example, if the intention is "to intimidate," an actor might stride over to another actor in a belligerent manner, stare him in the face, and verbally threaten him, using a menacing tone of voice. The most important aspect of this procedure is the reality with which it is executed. The actor does not try to merely look and sound intimidating but actually attempts to intimidate the other actor in the scene. Up to now, we have looked at this process globally, measuring the memory effects of all the aspects of active experiencing in tandem (e.g., H. Noice \& T. Noice, 1999; H. Noice et al., 2000; T. Noice \& H. Noice, 1997a, 1998). In the experiments reported here, we tried to tease out (as much as possible) the specific contribution of movement itself. The question is, in the example above, would the attempt to intimidate another character produce as much verbal retention using just the threatening stare and the menacing tone of voice? Or is the motivated movement from place to place (the belligerent stride) necessary to produce a memory effect? And if it is, do the movement and the verbal material have to be paired repeatedly over a long period of time, typical of professional theatrical engagements? And would specific speeches on which the participants moved be more memorable than those on which the same participants did not move? Furthermore, could these effects (previously observed only with professional actors) be demonstrated with novice actors or even with participants with no acting background whatsoever? Finally, what are the theoretical implications of such nonliteral movement effects?

Recently, Glenberg and his associates (e.g., Glenberg, 1997; Glenberg \& Robertson, 1999) have proposed an action-centered theory based on the notion of embodiment. According to this view, memory is for the purpose of enabling us to function in the physical world. In fact, in reviewing the enactment literature, Glenberg commented, "One interpretation of this finding [the SPT effect] is that memory specializes in embodied information" (p. 5). Thus, the theory of embodied cognition finds an intimate connection between action, meaning, and language.

Specifically, Glenberg (1997) proposed that the meaning of a situation is the set of potential actions available in that situation to the individual experiencing it. This set of actions is determined by integrating affordances and experiences in order to attain goals. Affordances are properties that are jointly determined by the physical characteristics of the objects and the biological characteristics of the people interacting with them. Thus, a pencil can afford writing, throwing, poking, and probing, but only to a type of animal (human or otherwise) that can grasp it in ways consistent with those affordances. For example, a pencil's affordances (and hence, what it means) are different for a dog (the pencil can be chewed), an infant (the pencil can be chewed, poked, and thrown), and a literate adult. Finally, affordances must be combined to accomplish goals. For example, if a goal is to recover a marble from under a couch, the poking and probing affordances of a pencil become the predominate components of the pencil's meaning in the situation, and those affor- dances must be meshed with those of the marble in order to recover the marble from under the couch. Experiences retrieved from memory can help to flesh out the affordances derived from perception. Thus, in the above example, if one remembers getting a nasty splinter from a particular pencil, that pencil is apt to be avoided when trying to recover the marble.

This view of memory would seem particularly relevant to our own studies that center on the cognitiveemotive-physiological processes of professional actors. If the findings of the present study show that nonactors can also benefit from the nonliteral movement effect previously found with actors, such a result would indeed appear to conform to an embodied cognition account of human memory.

\section{EXPERIMENT 1}

In this experiment, beginning acting students worked on material for $5 \mathrm{~min}$ in one of three conditions. In the first, they tried to get across the meaning of the material by using both bodily movement and verbal communication. In the second condition, they used verbal communication only. A third (control) group simply memorized the same text. If those who engaged in movement during the learning phase recalled more material than did those who communicated the meaning only verbally or controls who deliberately memorized the same material, it would suggest that movement is a vital component of the active-experiencing effect.

In professional theatre, the final movement patterns are arrived at after a great deal of preliminary work. In the first phase of this work, actors analyze the script to determine the intentions of the characters, using those intentions to discover how the characters would talk and move within the dramatic situation (T. Noice \& H. Noice, 1997b). Generally, the director then gives a blocking overview for the scene (i.e., where the actors enter, where the bulk of the scene is played, relative positioning to make relationships clear, etc.). Within this framework, the actors use appropriate body language and quality of movement to communicate the characters' intentions as they have gleaned them. The director then selectively edits these spontaneous movements to arrive at the final blocking, which is entered into the promptbook. The question is whether this self-generation of various aspects of performance is essential to the nonliteral movement effect or whether novices might receive the same memory benefits, assuming that an experienced director performed a preanalysis of an easily understood scene and then briefly instructed the novices in the execution of the necessary intention-fulfilling movements.

\section{Method}

Participants. Twenty-three undergraduates enrolled in Beginning Acting for Non-majors took part in the experiment as a course requirement. They were all native English speakers. Since the design called for students with no previous acting experience, 8 additional students were eliminated from the data analysis because their 
questionnaires revealed extensive acting experience outside of college. Thus, the remaining 23 students (10 males and 13 females) had no background in theatre, except for the first two-thirds of a course available only to non-theatre-majors to satisfy a general education requirement. The rationale for using these particular participants was that they had had enough instruction and practice in the technicalities of preparing a role to be comfortable in the experimental situation but did not possess any expertise derived from extensive practical experience.

Materials. A scene from the play The Dining Room by A. R. Gurney Jr. (1982) was used in this experiment. In this scene, two siblings argue over the dividing of their parents' furniture between them. One role consisted of 26 speeches, and the other of 22 speeches. Two booklets were prepared, one containing the scene itself and one containing a brief distractor task. This second booklet also contained a copy of the studied scene, in which all the assigned character's dialogue had been replaced by blank spaces. In addition, this booklet contained a very brief questionnaire asking whether the participant had had any acting experience other than the current course and whether he or she had ever read or seen the play The Dining Room.

Design and Procedure. All the participants were told to attend to one of the roles in this two-character scene and were assigned to one of three conditions: the full active-experiencing condition (with movement), the partial active-experiencing condition (verbal communication only), or the memorization condition (control group). Because the two experimental conditions required participants to work in pairs, the 23 participants were divided as follows: 8 in the full active-experiencing condition, 8 in the partial active experiencing condition, and 7 in the memorization condition. With the exception of this one constraint (assigning even numbers to the two experimental conditions), all the participants were randomly assigned to conditions, and all were allotted $5 \mathrm{~min}$ to process the dialogue.

This particular scene was chosen because the intentions of the characters were fairly apparent, making extensive preliminary discussion unnecessary. Furthermore, the movements (devised by a professional director) were clearly related to the dialogue and did not require lengthy explanation. For example, when one character tries to end a confrontation by leaving the room, the other character blocks the first character's way. The director devised the movement scheme so that both characters had a nearly equal number of movements and both characters had some speeches on which they moved and other speeches on which they did not.

Two at a time, those in the full active-experiencing condition first learned the pattern of movements about the room without having any idea of why they were executing those particular movements or of the dialogue that would eventually accompany them. The students were told that the reasons for the movements would become clear when they received the scripts with the dialogue. These participants were subsequently handed these scripts and told to read them aloud to each other while moving about as taught for exactly $5 \mathrm{~min}$. Although they had already learned the movement patterns in abstract, the scripts contained the usual theatre abbreviations for movement (e.g., "x u. of table, c." means "cross to the far side of the center table") inserted just before the speeches on which the movements were required. Because the participants had already had a couple of months of acting training, they had no trouble understanding the movement directions and were able to execute them with a certain amount of spontaneity. That is, each couple maintained the same general movement patterns but did not necessarily start and end every move mechanically on exactly the same word or syllable. Rather, each couple performed the overall movement scheme with minor individual variations.

The pairs of participants in the partial active-experiencing condition sat on chairs facing each other and read the dialogue aloud. The students in both experimental conditions were specifically instructed not to try to memorize the dialogue but to put all their concentration on meaning what they were saying, actively trying to communicate those meanings to their partners. (This concentration on genuine communication tends to give rise to affect states consistent with the dramatic situation and thus involves the emotive component of the active-experiencing principle.)

The 7 participants in the memorization condition did not perform the task in pairs. They were told to commit their roles to memory, using whatever procedures they had found to be successful in the past. Thus, the controls' learning strategies were not imposed by the experimenters but reflected the way these students normally learned material.

Prior to the manipulations themselves, the experimenters discussed the nature of research in general (but not of this experiment) with the participants in the partial active-experiencing and the memorization conditions. This took approximately the same amount of time that it took to teach those in the full active-experiencing condition the movement patterns and was done so that all the participants had the same amount of interaction with the experimenters and spent the same amount of total elapsed time, thus controlling for the possibility that such extraneous factors might have a bearing on the results.

At the end of the learning phase, the scripts were exchanged for recall booklets, which contained the distractor task (writing down the titles of five plays or movies they had seen recently and rating the appeal of each on a 5-point Likert scale), followed by a cuedrecall task for the entire studied role and the brief questionnaire. The recall booklet contained all the utterances of the "other" character in the scene, interspersed with blank spaces for the participants to write out their own speeches. All the students, regardless of condition, were seated during recall and worked by themselves. Although there was no time limit for the recall phase, all the students finished the whole experiment in under $30 \mathrm{~min}$. Upon completion, they were debriefed and thanked for their participation.

Scoring. The dependent measures used were a strict verbatim measure that gave credit only if a speech was recalled with absolute word-for-word accuracy and a slightly less stringent measure, almost verbatim, which accepted only precisely defined minor changes, such as using contractions instead of whole verb forms or substituting one exact synonym in an entire utterance. A third measure, close paraphrase, gave credit for any speech that did not meet the strict criteria for almost verbatim but that, nevertheless, contained both the exact idea and at least $50 \%$ of the original operative words. Verbatim and almost verbatim were combined to form the acceptable verbatim measure, and acceptable verbatim and close paraphrase were combined to form the total recall measure. Even if a participant's recall contained the same ideas as the original utterance, but with fewer than $50 \%$ of the original operative words, it was, for almost the purpose of this very strict scoring scheme, considered as not recalled. For example, if the original wording was "You mean you want two dining rooms" and the participant remembered it as "You would have two dining rooms then," that utterance would not be included in the recall results.

One rater scored all of the protocols, and a second independent rater scored a random subset of $30 \%$. Interrater reliability was $98 \%$ for verbatim, $88 \%$ for almost verbatim, and $83 \%$ for close paraphrases. Disagreements were resolved by mutual consent.

\section{Results and Discussion}

Protocols were analyzed with respect to the number of speeches recalled within our strict parameters. The mean proportions of speeches recalled under the first measure, true verbatim, were calculated and are presented in Table 1 (with the corresponding standard deviations).

A preliminary analysis revealed that there was no main effect or interaction that involved assigned role (sibling A vs. sibling B). We therefore collapsed over this factor in subsequent statistical tests. A one-way analysis 
Table 1

Mean Proportion of Speeches Recalled Verbatim for Experiments 1 and 2

\begin{tabular}{llllll}
\hline & \multicolumn{2}{c}{ Experiment 1 } & & \multicolumn{2}{c}{ Experiment 2 } \\
\cline { 2 - 3 } Testing Condition & $M$ & $S D$ & & $M$ & $S D$ \\
\hline $\begin{array}{l}\text { Full active experiencing } \\
\quad \text { (with movement) }\end{array}$ & .38 & .10 & & .31 & .14 \\
$\begin{array}{l}\text { Partial active experiencing } \\
\quad \text { (verbal communication only) }\end{array}$ & .21 & .18 & & .23 & .13 \\
Memorization & .14 & .04 & .19 & .11 \\
\hline
\end{tabular}

of variance (ANOVA) performed on the true verbatim data revealed a significant effect of learning strategy $\left[F(2,20)=7.66, M S_{\mathrm{e}}=0.02, p<.01\right]$. A Tukey post hoc test indicated that recall was higher for those acting students who had processed the scene while moving (full active experiencing), relative to acting students in the other two conditions, but that recall performance of students who used verbal communication only (partial active experiencing) was not superior to those who deliberately memorized the material.

Subsequent analyses using our second measure, acceptable verbatim, also found an advantage for the full active-experiencing condition over both the partial active-experiencing condition and the memorization condition $[M=.57$ vs. $M=.28$ vs. $M=.24 ; F(2,20)=$ $9.36 ; p<.01]$. This advantage also held for our third measure, total recall $[M=.76$ vs. $M=.46$ vs. $M=.37$; $F(2,20)=9.92, p<.01]$.

This superior recall of the text in the moving condition cannot be explained by extra exposure to the to-beremembered material during the preliminary training in the patterns of movement, because the participants had no idea of the dialogue or content of the scene when they were taught these patterns. The participants in all the conditions processed the material for exactly $5 \mathrm{~min}$. The postexperiment questionnaire revealed that none of the participants had ever seen or read the play.

The important finding here is that facilitation of verbal recall owing to accompanying nonliteral movement required neither expertise, nor self-generation of the intentional movements, nor repeated pairings of stimuli over leng thy periods. Students who actively experienced communicating the meaning of the material by using both words and movements recalled significantly more material than did either students who communicated using only words or students who deliberately memorized the same text. It is interesting that this memory enhancement was found even though these students had undoubtedly never paired these movements with these words before in their lives. By comparison, in a typical SPT experiment, the actions and the objects on which the actions are performed often contain a universal, lifelong mental association (e.g., throw the ball, cut the bread), although a few experiments (e.g., Engelkamp, Zimmer, \& Biegelmann, 1993; Mohr, Engelkamp, \& Zimmer, 1989) have shown that the SPT effect can also be obtained with "bizarre" phrases, such as "plant the hammer" or "comb the toothbrush." With such phrases, it is highly unlikely that the actions and the objects had ever been previously paired. However, in contrast to our procedures, participants in such experiments did perform the literal actions of planting a hammer or combing a toothbrush (with imaginary objects) before the usual SPT effect was obtained.

\section{EXPERIMENT 2}

Although the participants in Experiment 1 were undergraduates with no prior theatrical experience, they still had a certain amount of acting training (the first part of an introductory acting course, chosen to fulfill a general education requirement). This left open the question of whether these results could be replicated with a general college population with no theatre training whatsoever. If this were possible, the findings might have strong implications for pedagogy.

Moreover, it might be objected that the control group in Experiment 1, who learned the text by deliberately memorizing it while sitting by themselves, did not fully mirror the experimental groups in which the participants worked with partners and spoke the material aloud. Therefore, we devised a second experiment, one with a design that allowed us not only to replicate the first experiment under more stringent conditions, but also to investigate the effects of movement on verbal memory with much greater specificity. Of course, the differences in the amount of training possessed by the students in Experiments 1 and 2 were so minor that we would certainly not consider this a manipulation of expertise. It is frequently said (e.g., Meisner \& Longwell, 1987) that it takes about 20 years of experience to produce a truly skillful professional actor. Thus, the only real difference between groups was that the participants in Experiment 1 had had enough practice in class to eliminate some of the awkwardness that comes with a first attempt to "act" in public.

\section{Method}

Materials. The same two-character scene from the play The Dining Room was used so the results would be directly comparable. Since we had already demonstrated the existence of the nonliteral movement effect with a different play (H. Noice \& T. Noice, 1999), it was not necessary to provide evidence here that the effect would generalize to other types of material. The recall booklet also included a questionnaire asking the participants whether they had ever seen or read the play or whether they had had any acting experience in or out of college.

Participants. This time, the participants came from a general undergraduate population, who took part in the experiment for extra credit. None of them had taken even a basic theatre course or had any stage experience, except for chorus parts in high school. Fiftyfour students (none of whom had ever seen or read the play) were randomly assigned to one of three learning strategies.

Design and Procedure. The participants were tested in pairs. All the participants were again told to attend to one of the roles in this two-character scene and were randomly assigned to the full active-experiencing condition (with movement), the partial activeexperiencing condition (verbal communication only), or the mem- 
orization condition (control group). However, the control group used a different approach from that employed in Experiment 1. In Experiment 1, we had opted to use a procedure that seemed most representative of how students normally learn material. Therefore, we asked them to memorize the words as they would in their everyday lives. But in case there was some benefit to reading the dialogue aloud in pairs, we now had these controls also study aloud in pairs. However, they were specif ically told that this was a memory experiment and to put all their concentration into learning the exact text, not into "acting" the role.

The two experimental conditions remained the same: full active experiencing and partial active experiencing. The movement patterns for the former were the same as those for Experiment 1. These directions were inserted into the scene in standard printed-script format (i.e., in parentheses, before the dialogue that accompanies the movements). However, standard theatrical abbreviations were not used; for example, instead of "x u. 1. of table," these directions said "cross to behind the table from the leftside."

Even with these more explicit instructions, we anticipated that students with no acting training whatsoever might find the moving condition difficult to learn. Pilot testing confirmed that this was indeed the case. Although the students were shown the traffic patterns in advance, they found it extremely difficult, if not impossible, to coordinate words and movement when they received the actual scripts. To remedy this, we prepared special scripts with rows of Xs in place of the words. Thus, these "dummy" scripts contained directions on where and when to move and when to remain in place, but no dialogue. The students then practiced the procedure, counting aloud $(1,2,3,4,5$, etc.) as they moved about the room until they were comfortable talking aloud and following the written movement directions at the same time. Of course, we could instead have had the students say, "blah, blah, blah..." or recite some poem they knew, but counting aloud while moving seemed the most natural way to practice coordinating sound and movement, the one requiring the fewest attentional resources, and the one least likely to cause embarrassment. (Obviously we could not give the students in this condition the actual dialogue to practice with, since this would have allowed them more time with the to-be-learned material than those in the other conditions, introducing a confound.)

The preliminary movement training generally took less than $10 \mathrm{~min}$. The participants were then given the actual scripts with the dialogue and rehearsed the scene for exactly $5 \mathrm{~min}$, utilizing both words and movements. This procedure provided a great opportunity to investigate yet one more issue: the correspondence between recall of a speech and whether or not that speech had been accompanied by movement. The acting students in Experiment 1 had followed the same general movement patterns, but with a certain amount of individuality, depending on how they interpreted the scene. On the other hand, these non-acting-students in Experiment 2 rigorously followed the exact line-by-line format as set by the director, moving when and where indicated and not moving at all when the dialogue was prefaced by the notation: "stay." This allowed us to eventually assess the relationship between movement and verbal memory on a speech-by-speech basis.

All other aspects of the procedure, including the preliminary interaction with the participants in the partial active-experiencing and memorization conditions, were identical to those described for Experiment 1, as were the nature of the distractor task and the cuedrecall booklet.

\section{Results and Discussion}

The scoring scheme was identical to that used in Experiment 1 . One independent rater, blind with respect to the participant's condition, scored all of the verbal protocols, and a random subset of $30 \%$ was scored by a second rater. Any disagreements were resolved by discus- sion. Agreement between the two scorers was $99 \%$ for verbatim, $89 \%$ for acceptable verbatim, and $85 \%$ for close paraphrases.

Table 1 presents the mean proportion of speeches recalled verbatim as a function of learning condition-full active-experiencing condition (with movement), partial active-experiencing condition (verbal communication only), and memorization. Standard deviations are also shown. These data were analyzed using a two-factor (learning strategy and assigned role) between-subjects ANOVA. Since the assigned role variable did not interact with learning strategy, the data were collapsed over this factor. A follow-up ANOVA conducted on the speeches recalled verbatim revealed a significant effect of learning strategy $\left[F(2,51)=4.68, M S_{\mathrm{e}}=0.02, p<\right.$ .05]. A Tukey's HSD test indicated that recall in the full active-experiencing condition was higher relative to the memorization condition. However, it did not differ significantly from the partial active-experiencing condition, and neither did the recall of the partial active-experiencing group differ from that of the memorization group.

Similar analyses were performed on the other two dependent measures, acceptable verbatim and total recall. Since they yielded comparable effects, only the former will be reported. Again, a significant main effect of learning strategy was obtained $\left[F(2,51)=7.06, M S_{\mathrm{e}}=0.02\right.$, $p<.01]$. As in the previous analysis with true verbatim, a post hoc Tukey's HSD test confirmed that those participants who had moved during learning $(M=.46, S D=$ .11) outperformed those who had deliberately memorized the script $(M=.20, S D=.14)$.

We next addressed the question of whether those speeches on which the participants had moved were recalled better than those on which they had remained in place. As in the accuracy analysis, we counted only those utterances that had been categorized as verbatim, acceptable verbatim, or close paraphrase. These data were then transformed into percentages and analyzed in a mixed ANOVA, with two between-subjects variables, strategy (full active-experiencing vs. memorization) and assigned role (sibling A vs. sibling B), and one withinsubjects variable, type of line (lines performed while moving vs. lines performed while remaining in place). Table 2 presents the means and standard deviations, collapsed over assigned role.

There were significant main effects of strategy $[F(1,32)$ $\left.=12.22, M S_{\mathrm{e}}=551.83, p<.01\right]$ and type of speech $\left[F(1,32)=4.43, M S_{\mathrm{e}}=281.37, p<.05\right]$, which confirmed that recall was, in general, better for the full active-experiencing group than for the memorization group and that speeches accompanied by movement were better remembered than those performed without movement. No effect involving assigned role was significant (all $F \mathrm{~s}<1)$.

Most important for our purposes, however, was the significant interaction between learning strategy and type of speech $\left[F(1,32)=4.96, M S_{\mathrm{e}}=281.37, p<.05\right]$, which indicated that speeches accompanied by move- 
Table 2

Mean Percentages of Speeches Recalled as a Function of Movement

\begin{tabular}{|c|c|c|c|c|}
\hline \multirow{2}{*}{$\begin{array}{l}\text { Testing } \\
\text { Condition }\end{array}$} & \multicolumn{2}{|c|}{$\begin{array}{c}\text { Lines Spoken } \\
\text { With Movement }\end{array}$} & \multicolumn{2}{|c|}{$\begin{array}{c}\text { Lines Spoke } \\
\text { Without Movement } \\
\end{array}$} \\
\hline & $M$ & $S D$ & $M$ & $S D$ \\
\hline $\begin{array}{l}\text { Full active experiencing } \\
\text { (with movement) }\end{array}$ & 69.30 & 17.40 & 52.17 & 15.89 \\
\hline Memorization & 41.14 & 25.74 & 41.62 & 19.53 \\
\hline
\end{tabular}

ment were recalled significantly better than those without movement, but only by the full active-experiencing group. This finding underscores the contribution of nonliteral movement to verbal memory. The observed advantage could not have been due to those speeches being in themselves more memorable, because the memorization controls (who had no idea which speeches had been accompanied by movement in the moving condition) recalled an equal number of both types.

\section{GENERAL DISCUSSION}

These two experiments demonstrated that the effects of nonliteral movement previously found after lengthy real-world rehearsal and repeated performances by professional actors could be produced by giving a few minutes of instruction to nonactors with little or no performing experience. Although the results for Experiments 1 and 2 were generally quite similar, the differences that did emerge were more of degree than of kind. As compared with the acting students, the recall of the general college population was depressed in the full active-experiencing condition, but not in the memorization condition. Obviously, this finding is not due to real differences in expertise; a few weeks of training cannot produce experts. However, such training can reduce the tension and awkwardness of first-time performance and might account for the observed differences. The amount of recall by acting students in the full active-experiencing condition is particularly striking. They remembered approximately $76 \%$ (total recall) of the scene, as compared with $37 \%$ for the deliberate memorization controls, impressive evidence for the efficacy of the active-experiencing strategy.

One interesting point in the full active-experiencing condition concerns the large increases in recall from the true verbatim measure to the acceptable verbatim measure by both populations (from 38\% to 57\% in Experiment 1 and from $31 \%$ to $46 \%$ in Experiment 2), demonstrating extensive recall that just misses being flawless. This is not surprising in light of many of our discussions with professional actors. They have frequently stated that their natural strategy (the one we call active-experiencing) delivers most of the exact words but sometimes omits a word of secondary importance. For example, if a scripted line of dialogue is "When that girl is around, I just seem to fall apart," the actor might recall it as "When that girl is around, I seem to fall apart." When this happens, in order to ensure absolute word-for-word retention, a professional actor then would have to reanalyze the text to supply a reason why the character used the qualifier "just" in that utterance. Obviously, these novices had neither the time nor the expertise for such fine-tuning. Therefore, a number of almost perfectly recalled utterances had to be classified as acceptable verbatim instead of true verbatim, even though only a one-word qualifier was missing from the entire utterance.

We have previously speculated that some explanations offered for the SPT phenomenon may also apply to this nonliteral movement effect (H. Noice \& T. Noice, 1999). One group of researchers (e.g., Kormi-Nouri, 1995; Kormi-Nouri \& Nilsson, 1998) considers the SPT effect to be a result of distinctiveness owing to the integration of the verb and noun in the to-be-learned phrases. Because the accompanying phrases in our experiments were never literal and frequently did not contain verbs (e.g., "In a condominium?"), this explanation would not seem to fit our data.

However, those theories positing multimodal processing would not appear to conflict with the results reported here. For example, Engelkamp and Zimmer (1985) proposed that enactment results in the formation of separate codes for the verbal, visual, and motor modalities (with the latter providing strong item-specific processing), and Cohen (1989) suggested that enactment during original processing adds a motoric dimension to the memory trace. In a recent review, Nilsson (2000) compared the various theoretical positions of SPT investigators (e.g., Cohen, 1989; Engelkamp \& Zimmer, 1985; Nilsson \& Baeckman, 1989) and noted that (unlike Kormi-Nouri, 1995) a majority now emphasize both motor activity and nonstrategic processing. Our results might be interpreted as suggesting a motoric component in the nonliteral movement effect, and the nonstrategic aspect is particularly interesting in light of our demonstration of better memory for individual speeches on which participants moved, as compared with those on which they had remained in one place. Presumably, our participants were trying to communicate with their partners throughout the scene as they were instructed; yet, they remembered moving speeches better, suggesting that the extra encoding from movement occurs without strategic intent.

Obviously, the experimental procedures reported here have now moved much closer to those generally used to demonstrate the well-known enactment effect. That is, both paradigms now employ nonexpert participants who are given only very brief procedural instructions. However, some differences remain. In addition to the literal versus nonliteral variation, the experimental phrases in 
the majority of SPT studies involve a limited range of motion (lift the pen, move the ashtray, bend the paper-clip). Conversely, in our test scene, actions consisted primarily of gross body movements from one location to another. However, in the final part of our scene, one sibling stands still while holding up both hands with a small object concealed in one of them, in a final attempt to get the other sibling to pick one hand, thus determining who gets first choice of an item of furniture. This action is akin to those typically used by SPT researchers; yet, our participants in the moving condition did not recall the accompanying verbal statement ("I don't want to fight. Which hand?") as well as they recalled dialogue accompanied by a full body movement from one location to another. This discrepancy would have to be resolved before assuming that our movement-enhanced recall depends on the same mechanisms as those proposed to explain the typical SPT effect.

The theoretical position that would appear to best accommodate our data is that of embodied cognition, as advanced by Glenberg and his associates (e.g., Glenberg, 1997; Glenberg \& Robertson, 1999). In order to see how this position could explain the increased memorability we observed in the full active-experiencing condition, it is necessary to sketch in the embodied cognition view of language comprehension. Kaschak and Glenberg (2000) developed the three-process indexical hypothesis to explain how linguistic symbols become meaningful - that is, how language is used to change the set of actions available in a situation, thus changing the meaning of the situation. The first process is indexing words and phrases to objects and actions in the environment or to perceptual symbols (Barsalou, 1999). That is, the words are mapped to their referents. The second process is deriving affordances from the indexed objects. Finally, the meaning of a sentence is determined by meshing or integrating the affordances of various objects, as was discussed in our introduction. The meshing process is guided by goals arising from the general situation, as well as by goals specified by the syntactic structure of sentences. According to construction grammarians such as Goldberg (1995), the structures of simple sentences, or constructions, carry a general meaning over and above that carried by the words. According to Kaschak and Glenberg (2000), this general meaning is used to guide the mesh of affordances and thereby helps to determine the meaning of a sentence.

By this account, a sentence is understood to the extent that the affordances of the objects can be meshed to accomplish the goals specified by the sentence. For example, we can understand the sentence "Jack kissed Jill" because we can envision how the various affordances and goals can be meshed into smooth action. However, if the word "Jack" were indexed to a plaster statue, the meaning of the sentence would be obscure. Because language is used for coordinating action in the world, we must be able to smoothly integrate the meaning of situations in the world and the meaning of linguistic utterances. This can be done when both the concrete situations and the linguistic utterances are understood as possible sets of actions arising from the mesh of affordances.

It now remains to extend this theory to our data. That is, how does this view of language comprehension explain why our participants remembered dialogue uttered while moving better than dialogue uttered while remaining in one place? According to the indexical hypothesis, a sentence is comprehended when affordances are meshed in such a way that a specific meaning emerges. However, real movements by real actors provide concrete information that can communicate the meaning of accompanying dialogue beyond what the words alone convey. For example, if one actor walks up to the other slowly and hesitantly while saying "Can we please try it my way for a change?" the probable meaning is that the words constitute a plea from someone who habitually loses the argument. On the other hand, if the same line of dialogue is spoken by an actor who is walking toward the other in a strong, purposeful manner with head thrust forward, the probable meaning is more like a demand from someone who is tired of the other's wishy-washy ways. Thus, the quality, speed, and direction of the movements contribute a new source of affordances that mesh with the affordances provided by the indexed referents to supply a concrete meaning to the utterance "Can we please try it my way for a change?"

For another example, at one point in our test scene, sibling B walks to the other side of the room while saying "You mean you really want the dining room stuff?" The physical action of crossing from one part of a room to another could result from a number of possible affordances, including acquiring something that is at the other location, working off excess energy by pacing the floor, or, as in this case, avoiding a confrontation by walking away from the other person, thus stalling for time. Real actions (such as walking away from the other person) have properties that their prose descriptions would not. For example, they take different amounts of time and thereby change the actions available and the meaning. By crossing to the other side of the room, sibling B generates a time delay that, in this case, avoids a confrontation, adding greater specificity to the meaning (to stall) of the verbal utterance "You mean you really want the dining room stuff?" The statement and its underlying intention are literally embodied by the actor's movements. Such embodiment provides additional affordances, which increase the organization of the conceptualization and, therefore, enhance memory. It would appear that such an explanation would apply to both the standard SPT effect and the nonliteral effect reported here. In the case of the former, enactment adds concrete information to a phrase such as "open the book" by specifying how the book is opened (quickly? carefully?), its location (in the hands? on the table?), and so forth. With the nonliteral effect, enactment provides the same information but goes further by constraining dialogue that is typically far less concrete than the phrases used in most SPT research. We believe that this deep connection between meaning and 
action is responsible for the memory advantage we found in the full active-experiencing condition, a finding that would certainly follow logically from an embodied cognition account of memory.

To summarize, previous research (T. Noice \& H. Noice, 1997a, 1998) had shown that students who were told not to memorize but to actively experience, using all channels of communication (physical, emotive, and cognitive), retained more material (dramatic, narrative, or expository) than did controls who were told to deliberately memorize the same material. The experiments reported here demonstrated that the emotive and cognitive aspects (as performed in the partial active-experiencing condition) were not sufficiently powerful in themselves to create memory enhancement and that the addition of physical movement was crucial to the effect. (Of course, it is not possible to completely isolate the cognitive, emotive, and physiological aspects of human performance. Many studies [e.g., Berkowitz \& Troccoli, 1990; Bloch, 1989; Ekman, 1992] have shown that physical movements, even in the absence of specific contexts, tend to give rise to some affective experience. In actuality, our two activeexperiencing conditions were designed to investigate the additive effect of movement from place to place, over and above the effects produced by nonmoving emotive processing that used tone of voice, facial expression, and other means of communication.)

Thus, we have now been able to demonstrate this nonliteral movement effect with two more populations under circumstances widely different from those in the original demonstrations (H. Noice \& T. Noice, 1999; H. Noice et al., 2000), thereby increasing our confidence that the phenomenon is both genuine and robust. Finally, we have taken some tentative steps toward integrating these results into existing theoretical frameworks, although much additional investigation is necessary before one can use the nonliteral movement effect to definitively test competing theories of action-enhanced memory.

\section{REFERENCES}

Barsalou, L. W. (1999). Perceptual symbol systems. Behavioral \& Brain Sciences, 22, 577-660.

Berkowitz, L., \& Troccoli, B. T. (1990). Feelings, direction of attention and expressed evaluations of others. Cognition \& Emotion, 4, 305-325.

BLoch, S. (1989). Effector patterns of basic human emotions: An experimental model for emotional induction [Abstract]. Behavioral Brain Research, 33, 317.

Cohen, R. L. (1989). Memory for action events: The power of enactment. Educational Psychology Review, 1, 57-80

Ekman, P. (1992). Facial expressions of emotion: New findings, new questions. Psychological Science, 3, 34-38.

ENGELKAMP, J. (1991). Memory of action events: Some implications for memory theory and for imagery. In C. Cornoldi \& M. A. McDaniel (Eds.), Imagery and cognition (pp. 183-219). New York: Springer-Verlag.

Engelkamp, J. (1998). Memory for actions. Hove, U.K.: Psychology Press.

ENGELKAMP, J., \& ZiMmeR, H. D. (1985). Motor programs and their relation to semantic memory. German Journal of Psychology, 9, 239-254.

ENGElKAMP, J., \& ZimmeR, H. D. (1990). Memory for action events: A new field of research. Psychological Research, 51, 153-157.

ENGElKamp, J., \& Zimmer, H. D. (1994). The human memory: A multimodal approach. Seattle: Hogrefe \& Huber.

Engelkamp, J., Zimmer, H. D., \& Biegelmann, U. E. (1993). Bizarreness effects in verbal tasks and subject-performed tasks. European Journal of Cognitive Psychology, 5, 393-415.

GlenBerg, A. M. (1997). What memory is for. Behavioral \& Brain Sciences, 20, 1-55.

Glenberg, A. M., \& Robertson, D. A. (1999). Indexical understanding of instructions. Discourse Processes, 28, 1-26.

GoldBerg, A. E. (1995). Constructions: A construction grammar approach to argument structure. Chicago: University of Chicago Press.

Gurney, A. R., JR. (1982). The dining room. Garden City, NY: Doubleday.

KaschaK, M. P., \& Glenberg, A. M. (2000). Constructing meaning: The role of affordances and grammatical constructions in sentence comprehension. Journal of Memory \& Language, 43, 508-529.

Kormi-Nouri, R. (1995). The nature of memory for action events: An episodic integration view. European Journal of Cognitive Psychology, 7, 337-363.

Kormi-Nouri, R., \& NiLsSON, L.-G. (1998). The role of integration in recognition failure and action memory. Memory \& Cognition, 26 , 681-691.

MEISNER, S., \& LongWell. D. (1987). Sanford Meisner on acting. New York: Vintage Books.

Mohr, G., EngelKamp, J., \& Zimmer, H. D. (1989). Recall and recognition of self-performed acts. Psychological Research, 51, 181-187.

Nilsson, L.-G. (2000). Remembering actions and words. In E. Tulving \& F. I. M. Craik (Eds.), The Oxford handbook of memory (pp. 137148). New York: Oxford University Press.

NiLsson, L.-G., \& BäcKMAN, L. (1989). Implicit memory and the enactment of verbal instructions. In S. Lewandowsky, J. Dunn, \& K. Kirsner (Eds.), Implicit memory: Theoretical issues (pp. 173-183). Hillsdale, NJ: Erlbaum.

Noice, H., \& Noice, T. (1993). The effects of segmentation on the recall of theatrical material. Poetics, 22, 51-67.

Noice, H., \& NoICE T. (1994). Analyzing the role preparation of a professional actor. Discourse Processes, 18, 345-369.

Noice, H., \& Noice, T. (1999). Long-term retention of theatrical roles. Memory, 7, 357-382.

Noice, H., Noice, T., \& Kennedy, C. (2000). Effects of enactment by professional actors at encoding and retrieval. Memory, 9, 353-363.

Noice, T., \& Nolce, H. (1997a). Effort and active-experiencing as factors in verbatim recall. Discourse Processes, 23, 51-69.

Noice, T., \& Noice, H. (1997b). Expertise of professional actors: A cognitive view. Hillsdale, NJ: Erlbaum.

Noice, T., \& Noice, H. (1998, July). Memory benefits of activeexperiencing for expository and narrative material. Paper presented at the Eighth Annual Meeting of the Society for Text and Discourse, Madison, WI.

(Manuscript received September 27, 2000; revision accepted for publication May 5, 2001.) 